\title{
European Paediatric Formulation Initiative Workshop Report: Improving the Administration of Oral Liquid Medicines in Paediatrics using Dosing Syringes and Enteral Accessories
}

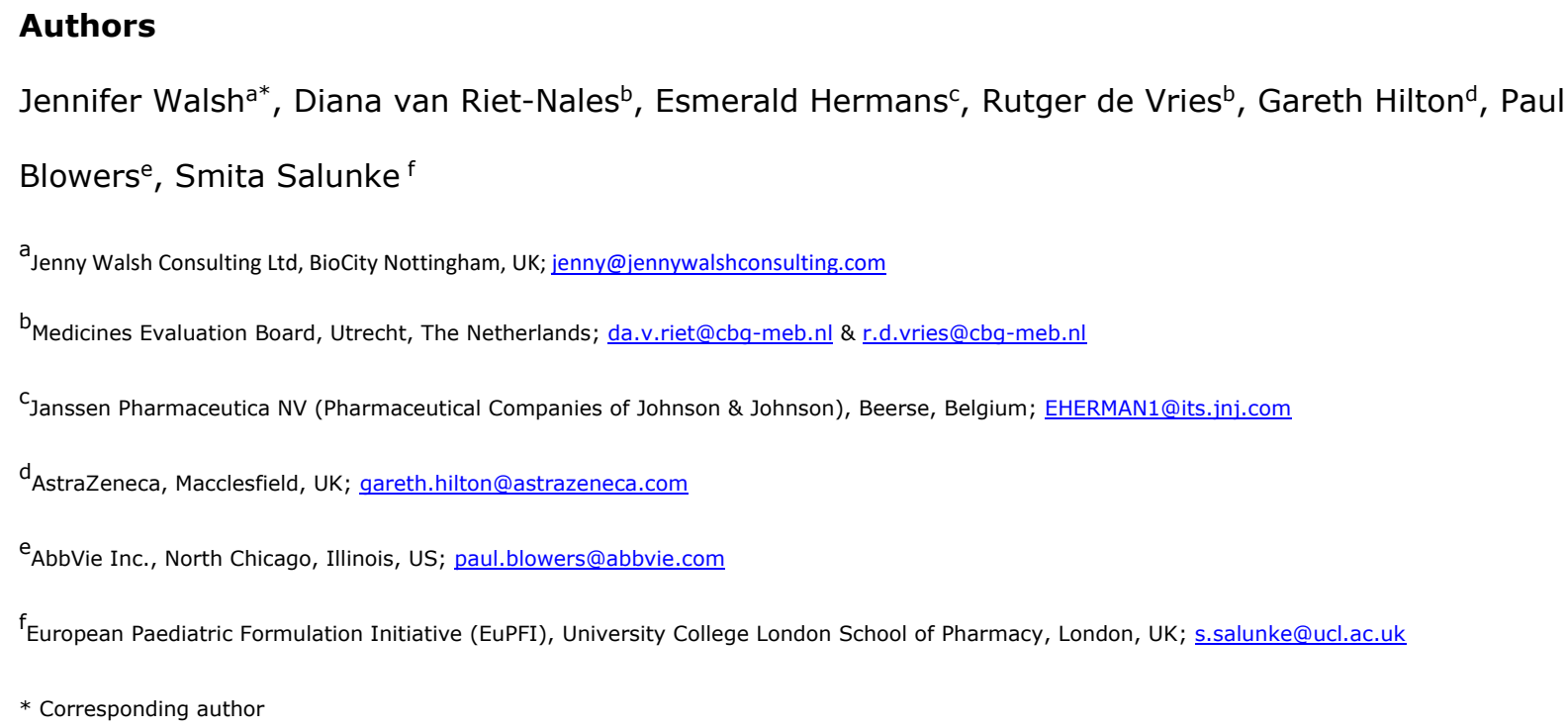

\section{Conflict of interest (at time of conference)}

Rutger de Vries \& Diana van Riet-Nales are members of the European Medicines Agency (EMA) Paediatric Committee (PDCO) Formulation Working Group (FWG). Diana van Riet-Nales is also member of the EMA Quality Working Party and the Dutch Medicines Evaluation Board (MEB) Committee on Clinical Practice.

Diana van Riet-Nales was rapporteur for the EMA Guideline on pharmaceutical development of medicines for paediatric use, the reflection paper on the pharmaceutical development of medicines for use in the older population and the EMA Q\&A on small volumes. She was a drafting group member of the Q\&A on enteral feeding and the EMA guideline on drug device combination products. Rutger de Vries supported the work on the paediatric guideline. He was a member of the drafting group on the EMA Q\&A for small volumes and enteral feeding.

Jennifer Walsh, Esmerald Hermans, Gareth Hilton, Paul Blowers and Smita Salunke are members of the European Paediatric Formulation Initiative. 
This Workshop did not receive any specific grant from funding agencies in the public, commercial, or not-for-profit sectors. None of the authors or speakers received any compensation or funding for the Workshop or manuscript publication.

\section{Disclaimer}

The views expressed in this article either represent the majority opinion of the workshop participants or they are the personal views of the authors. All views may not be understood or quoted as being made on behalf of or reflecting the position of their employers including the EMA, MEB, or any of its committees or working parties.

\section{Keywords}

- $\quad$ Drug development

- Medical device

- Infant, newborn

- Medication error

- Medication safety

- Administration, oral

- $\quad$ Dosing accuracy

- $\quad$ EMA

- Paediatric 


\section{ABSTRACT}

Accurate dosing of the right medicine to the right patient is a key element of safe and efficacious pharmacotherapy, yet prone to technical challenges and human error when dosing involves the administration of small volumes of liquid medicines. For this reason, the topic has gained increased attention over the last decade from multiple stakeholder parties e.g. academia, hospital pharmacy, the medical device and pharmaceutical industry, and regulatory agencies. It is now well acknowledged that spoons and cups are not suitable for the measurement of small volumes of oral liquid medicines and that syringes are a better alternative, but syringes for parenteral use should not be used for oral dosing in order to avoid accidental parenteral delivery of oral products. However, dosing accuracy of very small volumes of liquid medicines to young children, and especially pre-term neonates, is still not sufficiently ensured. A workshop was organised by the European Paediatric Formulation Initiative to reflect on current status and challenges (first part) and possible strategies to improve the present situation (second part). A voting system $(n=24)$ was used to consider the most favourable solutions. The harmonisation and/or standardisation of the technical design of oral syringes (including e.g. female/male connection) was considered a key priority. 


\section{Introduction}

Adequate medical care to neonates and infants involves an ongoing challenge to enhance overall child survival rates, reduce temporary or life-long disabilities, and encourage mother child bonding [1]. Although paediatric regulations have had a positive impact on the development of medicines [2], the availability of authorised, age-appropriate and affordable medicines for children is still limited, especially for off-patent drugs [3-5]. This situation worsens with decreasing patient age [3, 6]. Moreover, the younger the child, the greater impact suboptimal pharmacotherapy may have on health outcomes with potentially life long associated costs for medical care [7].

Neonates obtain most, if not all, of their medicines as small volumes of liquid medicines, either orally or parenterally as a result of disease status or immature swallowing capabilities $[6,8]$. Drug delivery can be challenging when children need medicines that are only authorised for adult use, especially when small volumes are required due to the products concentration e.g. $<0.1 \mathrm{~mL}$ [7] . Currently available devices may not have acceptable accuracy for the measurement of such volumes, and prior dilution of the available product to increase the volume to be measured and hence the accuracy of dosing can lead to calculation and/or manipulation errors, as well as the need to ensure any diluents used are compatible with the drug product [9]. The oral syringe is considered to be the measuring device of choice for oral liquids, with high dosing accuracy and uniformity, especially when small and accurate volumes are required $[10,11]$. However, it is important that the design and size of the syringe is appropriate for the volume to be measured to reduce the risk of dose variability [12]. Mis-dosing may occur with small volumes for example when an unsuitable size generic dosing syringe is used or when a drug specific device is unintentionally used for a different product. In addition, medication errors may occur when graduation in $\mathrm{mg}$ product or $\mathrm{kg}$ bodyweight is mixed with graduation in $\mathrm{mLs}$ or the graduation is not read in the way it should. Indeed, it has been reported that patients may have difficulties in identifying and/or measuring the correct dose when using oral syringes [13].

A new global enteral feeding device connector has been designed that complies with international standard ISO 80369-3, together with a range of compatible accessories and enteral syringes (commonly known as "ENFit") [14]. This change has been implemented to ensure all enteral (tube feeding) devices are specific to tube feeding, to avoid misconnections with for example intravenous (IV) systems [14]. Every extension set, enteral syringe and naso-gastric (NG) tube is 
designed with a specific "ENFit" end so that it is only possible to use products that are designed for enteral/tube feeding access. Hence, syringes for enteral feeding incorporate a female (wide) connector end that connects to the male connector of the feeding port. However, it may be necessary to use several adaptors to accurately measure and administer medication using such syringes in clinical practice which can be challenging and potentially lead to dosing errors.

In 2007, the European Paediatric Formulation Initiative (EuPFI) was formed to support global incentives to foster the development of authorised and age-appropriate medicines for children and to resolve scientific, technical and regulatory problems. The consortium has members from the pharmaceutical industry, hospitals and academia, as well as sponsors and subject matter experts, and the European Medicines Agency (EMA) as an observer. Its mission is to promote and facilitate preparation of better and safer medicines for children through interactive discussion between industry, academia, clinical and regulatory professionals, information dissemination (publications, conferences etc.) and collaboration and networking. There are five workstreams, one of which is focussed on paediatric administration devices [15].

In 2018 , a preconference workshop to the annual congress was dedicated to improving the administration of small volume oral medicines using dosing syringes and enteral accessories. The aims of the workshop were to determine and review stakeholder current practices, challenges and issues regarding oral dosing of liquid drug products to paediatrics, and to identify potential strategies to facilitate accurate and easy dosing that meet the needs of patients and the requirements of regulatory agencies, industry and suppliers. This workshop paper provides an overview of the presentations, team discussion, pending issues and recommendations for future incentives.

\section{Method}

Details of the workshop were shared via EuPFI contacts, professional bodies, social media, and patient groups. Workshop participants were selected through submitted Expression of Interest Forms to ensure appropriate representation from various stakeholder groups. Since a key aim of the workshop was to find solutions to resolve identified problems, only participants with experience of designing, testing, supplying, using or assessing oral liquid drug product dosing devices were permitted to attend. In addition, participant numbers were limited to encourage engagement in the 
team discussions. Pre-reading material including key dosing device guidelines, regulations, published research and other relevant provisions were shared with participants before the event.

The workshop was structured to facilitate the sharing of information and open dialogue between the different participants. The first half of the workshop comprised two plenary sessions. The first session included presentations on oral dosing device challenges and potential solutions given by healthcare professionals (a paediatric pharmacist, a paediatric clinical trials pharmacist, a paediatrician and a hospital medicines management nurse), and representatives from the pharmaceutical industry (a device engineer) and oral dosing device manufacturers. In the second session speakers from the Dutch and UK Regulatory agencies provided an overview of regulatory guidance and common questions and answers pertaining to the administration of oral liquid medicines. There was an opportunity for audience questions and answers after each speaker.

The second half of the workshop provided an opportunity for participants to discuss, present and appraise potential solutions. Participants were divided into 5 teams such that each team had balanced representation from each key stakeholder group and were presented with the following challenge and solution statements:

\section{Challenge:}

- Which procedural and operational challenges in relation to the administration of oral liquid medicinal products to the paediatric population in both a hospital and domiciliary setting (dosing devices, dosing accuracy etc) should be addressed?

\section{Solution:}

- What are constructive solutions that could facilitate the establishment of an optimal path towards the correct oral dosing of all paediatric patients with dosing syringes and enteral accessories, that meets the requirements of all stakeholders involved?

Each team was given 90 minutes to discuss challenges and then draw up one or more solution proposals onto flip charts before being given 5 minutes to present back to the other teams. Dot voting was then used to ascertain the participants' preferences for the proposed solutions. Each attendee was given 3 adhesive labels. Labels were colour coded to indicate which stakeholder 
group each attendee belonged to. Attendees were instructed to place their adhesive labels next to their preferred solution or solutions. The workshop closed with a summary of the day's discussions and the initial conclusions drawn from the voting process.

\section{Results}

\section{Participants}

Thirty-three participants from seven countries (Belgium, France, Germany, Netherlands, Switzerland, UK and USA), including the workshop organisers, attended the whole day, with an additional four participants attending for the morning session only. Approximately one third of participants were from the pharmaceutical industry $(n=12,37 \%)$ and regulatory agencies $(n=11$, $33 \%)$ respectively, whilst the remaining participants were healthcare professionals $(n=5,15 \%)$ or oral devices manufacturers $(n=5,15 \%)$. No patients or patient representatives attended despite being invited.

\section{Presentations}

\section{Healthcare professional perspectives}

Some of the challenges faced when administering oral liquid medicines to children with enteral syringes have been illustrated through recent research [16]. In this study, a comparison of generic oral dosing syringe brands indicated differences in dosing accuracy across brands and size of syringes, and those with a wide tip (female design) showed a greater dead space volume compared to those with a narrow tip (male design) across each syringe size (Figure 1). In addition, physical characteristics of the oral liquid had an impact on the dosing accuracy of the devices. None of the oral syringes were found to have acceptable accuracy for measuring volumes $<0.25 \mathrm{~mL}$, although neonates and infants often require the administration of small volumes. 
Figure 1 Syringe tip designs (reproduced with permission from GEDSA)

a) Male design

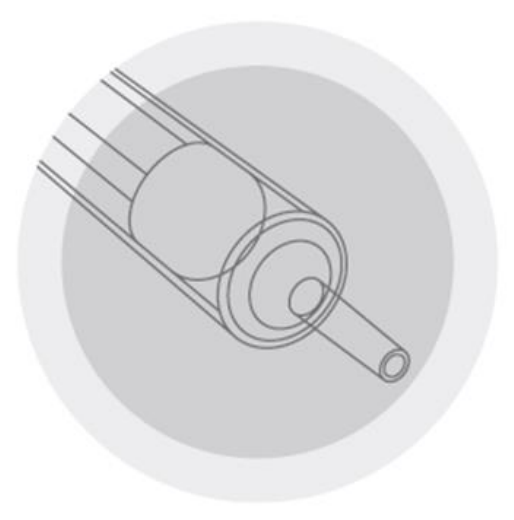

b) Female design (enteral)

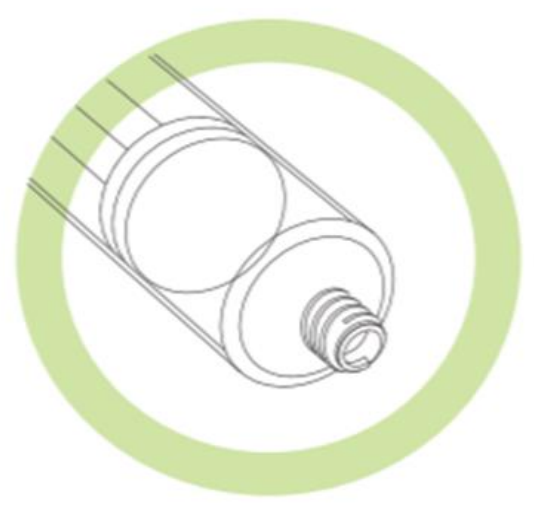

All of the above observations could lead to incorrect dosing and subsequently result in adverse events and/or lack of efficacy. The study demonstrated that some dosing syringe brands are not interchangeable, and that the size of the syringe is important. The researchers recommended that guidance is developed to advise which syringe size should be used for which dosing volumes. Furthermore, it was proposed that syringe designs should be consistent.

Oral and/or enteral syringes are required in paediatric clinical studies since oral liquids remain an appropriate formulation for many paediatric drug products in development. All administration devices used must comply with Regulatory Authority regulations, ISO and local (national) standards. However, a key challenge is the differing legal frameworks and standards across the globe which cause significant issues in terms of delays and complexity for multi-site international studies. Devices may be sourced locally in each country by the study site or centrally by the study sponsor, however there are different advantages and disadvantages to each approach, as 
summarised in Table 1. For example, although centrally sourced devices will have been tested for drug product compatibility and dosing accuracy, they may be incompatible with local (enteral) feeding tube connectors or locally used bottle adaptors. Accurate dosing, compatibility of the device with the product and safety are of key importance and transparency in and harmonisation of dosing syringe performance criteria are required.

Identifying and administering the correct dose of oral liquid medicines to paediatrics are key challenges. There are particular concerns for off-patent drugs for which an age-appropriate formulation may not be available, leading to the potential need to manipulate the adult dosage form prior to administration, which may have an unknown impact on the consistent drug product quality and/or bioavailability [17]. In addition, drug product compatibility with enteral feeding tubes and the determination of acceptable flush volumes are important. Some of the challenges faced when using oral dosing syringes include different units of measure (e.g. $\mathrm{mL}, \mathrm{mg}$, $\mathrm{kg}$ body weight) and graduations being removed after syringe washing. The use of units of measure by body weight for a product-specific syringe can cause mis-dosing when the same syringe is used for a different concentration of the same drug or a different drug product (despite the name of the original product being depicted on the syringe). Potential solutions to these problems include standard syringes with clear units of measure and the provision of comprehensive information on the correct use of the syringes. 
Table 1 Comparison of centrally and locally sourced oral dosing syringes for paediatric clinical studies

\begin{tabular}{|l|l|l|l|}
\hline \multicolumn{2}{|c|}{ Centrally Sourced (by Study Sponsor) } & \multicolumn{2}{c|}{ Locally Sourced (by Study Site) } \\
\hline \multicolumn{1}{|c|}{ Advantages } & \multicolumn{1}{|c|}{ Disadvantages } & Advantages & Disadvantages \\
\hline - Single supply & • May not meet local practices & $\bullet$ Meets local practices standards & $\bullet$ Many types and brands used \\
- Tested by Sponsor for use with & standards: & & Not tested for use with drug \\
drug product under & $\circ$ Non-CE marked & $\circ$ Unknown compatibility and \\
investigation: & $\circ$ Incompatible with feeding tube & & accuracy of dosing \\
$\circ$ Compatibility/stability & connectors (enteral) & \\
$\circ$ Accuracy and precision of & $\circ$ Ambiguous graduations & & \\
dosing & $\circ$ Incompatible in situ bottle & & \\
& adaptor & & \\
\hline
\end{tabular}


The new design enteral (ENFit) syringes and accessories are used for the administration of oral liquid medicines in hospitals to avoid the potential for mis-connection errors [14]. There is a huge variety in the design of oral dosing syringes provided with each drug product, and it is considered not practical to have oral and enteral dosing systems. Hence only enteral devices are used on the wards and then supplied to patients on hospital discharge. In order to achieve accuracy with the ENFit syringes it is necessary to use additional devices, for example bottle adaptors, or straws if the bottle adaptor is incompatible with the drug product bottle dimensions. This adds complexity to the medicine dosing process with increased risk of mis-dosing and requires specialist knowledge and training of the caregiver. In addition, there are significant cost implications associated with the purchase of the accessories and discarding the devices that are supplied with each product. Potential solutions include the standardisation of all oral and enteral syringes as well as drug product bottle neck dimensions. In addition, all commercial drug products could be supplied fitted with a bottle adaptor and compatible ISO standard enteral/oral syringe.

\section{Pharma Industry Perspectives}

The efficacy and safety of drug products have always been the focus of new drug product developments for both adult and paediatric patient groups. When developing for paediatric patients, in the past a liquid formulation used to be the galenic form of choice and the liquid was delivered by a measuring cup or spoon [18]. More recently, following dosing accuracy studies of over the counter products, the FDA has set stricter guidelines for dosing accuracy, while the EMA has provided reflections on typical oral dose volumes for different age groups [19], and subsequently general guidance on appropriate measuring devices for oral liquids [12]. These requirements effectively stop the use of dosing cups for volumes below $5 \mathrm{~mL}$ since evidence from studies suggests that it is somewhat difficult to dose small volumes correctly with cups, and to a lesser extent, with spoons $[10,18,20]$. Therefore, appropriately graduated oral dosing syringes are currently the preferred device for small volumes, although the situation may not be optimal yet [9]. In addition to dosing accuracy, ease of use and patient and caregiver engagement are key aspects of the device development process, to ensure that a therapy can be followed consistently [21]. A patient centric approach to paediatric drug product development is taken, leveraging the understanding of the caregiver and patient and linking clinical knowledge with desirable attributes of the drug product and its ease of dosing. To achieve this understanding, the needs and requirements of the patients are charted and linked to drug product requirements, resulting in 
concepts and solutions which merge all aspects, and which are evaluated during human factors studies $[22,23]$. This approach results in drug product specific oral dosing syringes which are easy to use for caregivers in varying environments and which comply with the stringent regulatory dosing accuracy requirements.

\section{Dosing Syringe Supplier Perspectives}

Dosing devices for medicines have been regulated in the EU since 1993 by the medical device directive 93/42/EC, now superseded by regulation EC/2017/745. A CE number is given to each device after auditing and approval by an accredited institute as a mark that the device complies with the regulation. Suppliers must provide complete device documentation such as specifications, declarations of compliance and information regarding the functionality of the dosing device. A justification of the intended use for which the CE mark is provided, is also discussed with customers.

There are currently no specific international dosing accuracy standards for oral dosing syringes, and therefore, compliance to ISO 7886-1, a standard developed for hypodermic syringes is fundamental to demonstrate the accuracy of oral syringes. The ISO 7886-1 standard also describes the required tolerances on the graduated capacities for each size of syringe and volume taken. Syringes complying with this ISO standard will comply to the PhEur and USP dosing accuracy requirements for oral liquids. Neonates, paediatric patients and adults are all at risk of medication related harm with unintended dose variability. However, the small doses and sometimes high concentrations which are given to neonates and small paediatric patients put these groups at high risk for dosage error. The need for accuracy is therefore very high in this patient group and clinicians should evaluate whether the device used provides to correct dose as labelled.

\section{Regulatory Agency Perspectives}

The challenge to administer small volumes has also been considered by the EMA Quality Working Party (QWP), and a new Q\&A document was being developed in response to discussions within/between different stakeholder parties, e.g. medical doctors, pharmacists, nurses, regulators, HTA-experts, device and the pharmaceutical industry. 
Key discussion topics included:

- The minimum volume that can be used for accurate dosing and whether this volume would depend on the type of setting (e.g. administration in the home setting, in neonatal care);

- What the minimum commonly available syringe size in Europe might be;

- Whether it would be appropriate to measure liquid medicines (other than insulin) with an insulin syringe (graduated in insulin units rather than $\mathrm{mL}$ ).

The practice of testing oral syringes against the requirements of ISO-7886-1, (the standard for hypodermic syringes), due to a lack of ISO standard for oral only syringes is recognised by Regulatory Authorities. The risk of wrong route administration errors, for example injecting oral or enteral products if prepared in parenteral syringes is a concern, and ideally oral syringes should be used for oral delivery and enteral (ENFit) syringes for connecting to enteral tubes. The different (wider) tip design of enteral syringes can have an impact on volume of liquid delivered and an enteral low dose tip syringe has been introduced to increase the dosing accuracy of small dose volumes. It should be noted that ISO standard $80369-3$ is applicable to the enteral connectors, but not enteral syringes. In order to reduce the risk of dosing and wrong route errors it is recommended that the connectivity of the equipment is checked, pharmacy dispense oral liquid medicines in oral syringes, staff awareness should be improved, and training programmes established.

\section{Challenges and Solutions}

The team discussion process identified numerous challenges associated with the correct oral dosing of small volumes, as shown in Table 2. In addition, multiple suggestions for addressing the issues were proposed. Voting results according to stakeholder group on potential solutions are presented in Table 3. Overall, the standardisation/harmonisation of oral and enteral syringes together with improving the accuracy of syringes for dosing small volumes were the highestscoring potential solutions. 
Table 2 Challenges associated with the correct oral dosing of small liquid volumes identified during team discussions.

How to design the most intuitive device for its intended use

Device development is stagnant compared to drug product development

Off-label use of devices for oral liquids

Non-universal (global) system for connectors

Lack of harmonisation regarding oral syringes leading to numerous devices and variability in design

Syringes are not standardised and so it is not always appropriate to use an "off the shelf" syringe for all products

Lack of dose accuracy, especially for the measurement of small liquid volumes

Table 3.

List of potential solutions to address issues with small volume liquid dosing proposed during team discussions and stakeholder voting results.

\begin{tabular}{|l|c|c|c|c|c|}
\hline \multirow{2}{*}{ Potential Solutions } & \multicolumn{3}{|c|}{ Number of Stakeholder Votes for each Potential } \\
& \multicolumn{5}{|c|}{ Solution } \\
\cline { 2 - 6 } & Industry & Regulatory & HCPs & Device & Total \\
\hline Standardisation of syringe design & 7 & 4 & & 2 & 13 \\
\hline Harmonisation of oral syringes & & & & & \\
worldwide; establish a standard to & & & & & \\
reduce variability of design and ISO & 8 & 3 & 1 & & 12 \\
\hline
\end{tabular}




\begin{tabular}{|c|c|c|c|c|c|}
\hline $\begin{array}{l}7886-1 \text { to include oral and enteral } \\
\text { syringes }\end{array}$ & & & & & \\
\hline $\begin{array}{l}\text { Improve accuracy of measuring small } \\
\text { volumes }\end{array}$ & 4 & 3 & 1 & 3 & 11 \\
\hline $\begin{array}{l}\text { Pharma to consider standard enteral } \\
\text { syringes and adaptors with standard } \\
\text { bottle necks for use at home and } \\
\text { hospital }\end{array}$ & 2 & 5 & 1 & & 8 \\
\hline $\begin{array}{l}\text { Device designers to visit clinical staff } \\
\text { and vice versa }\end{array}$ & 1 & 2 & 2 & 1 & 6 \\
\hline $\begin{array}{l}\text { Multi-disciplinary approach to reach } \\
\text { out to ISO } 20695 \text { specifying needs of } \\
\text { different stakeholders }\end{array}$ & & 2 & & 3 & 5 \\
\hline $\begin{array}{l}\text { CE mark to include the need to comply } \\
\text { with ISO standard for accuracy }\end{array}$ & 1 & & & 4 & 5 \\
\hline $\begin{array}{l}\text { Start syringe design with the hardest } \\
\text { patients in mind - neonates and } \\
\text { adolescents ( } 4 \mathrm{Kg} \text { cut-off and total of } \\
5 \mathrm{~mL} \text { per day for all drug products) }\end{array}$ & & 3 & & & 3 \\
\hline Study current best practice & 1 & & & 1 & 2 \\
\hline $\begin{array}{l}\text { Standardisation of adaptors and tip } \\
\text { design including fill technique multi vs } \\
\text { single use }\end{array}$ & & 1 & & 1 & 2 \\
\hline $0.5 \mathrm{~mL}$ syringe orphan device directive & & 1 & 1 & & 2 \\
\hline $\begin{array}{l}\text { Define minimum volume to be } \\
\text { measured with no markings below this } \\
\text { and accuracy data to be available. }\end{array}$ & 1 & & & & 1 \\
\hline
\end{tabular}




\begin{tabular}{|c|c|c|}
\hline $\begin{array}{l}\text { Optimise design of enteral and oral, } \\
\text { bottle + adaptor }\end{array}$ & 1 & 1 \\
\hline $\begin{array}{l}\text { Address off label syringes for liquid } \\
\text { medicines }\end{array}$ & 1 & 1 \\
\hline $\begin{array}{l}\text { Printing durability throughout cleaning } \\
\text { and maintenance }\end{array}$ & & 0 \\
\hline
\end{tabular}

HCP; healthcare professional.

Each participant was permitted to cast three votes. Some participants did not vote, including the workshop organisers.

Industry $n=8$, Regulatory $n=9$, HCPs $n=2$, Device Suppliers $n=5$

\section{Discussion}

The workshop provided an opportunity for direct dialogue between Regulators, HCPs (Clinicians, Nurses and Pharmacists), Industry and Device manufacturers/suppliers, and for participants to learn about other stakeholders' perspectives. The current practice of hospital HCP's preference for using off-the-shelf dosing devices instead of those provided by the Marketing Authorisation (MA) holder with the medicinal product was of particular interest to Industry, Regulators and Device suppliers. Furthermore, there was a lack of awareness of some of the day to day challenges faced by HCPs when dosing oral liquids to paediatrics, especially the low volumes commonly required for neonates and infants.

There was some variability in scoring of potential solutions according to stakeholder group. Although representatives from Industry and Regulatory agencies formed the largest groups, and not all participants voted, overall, the highest scoring potential solution was standardisation of oral and enteral dosing syringes followed closely by global harmonisation of oral syringes with the establishment of an ISO standard. Improvement in the accuracy of the measurement of small volumes also scored highly with votes from all four stakeholder groups. Interestingly, the proposal for Industry to consider standard enteral syringes and adaptors as standard with standard bottle necks for use at home and hospital scored highly amongst Regulators, and the CE mark to include the need to comply with an ISO standard for accuracy scored highly with the Device suppliers. 
Oral dosing syringes are considered to be more accurate for delivering small liquid volumes compared to other oral dosing devices $[10,11,18,24]$. As discussed during the workshop, syringe size and design as well as the physical properties of the oral liquid itself may have an impact on dosing accuracy [16]. Indeed, correct oral (into the mouth) dosing using enteral syringes (female tips) can be challenging and prone to error, especially when the design of the drug product bottle is such that additional accessories such as a bottle adaptor and/or medicine straw are required. The development and global introduction of the new ENFit enteral connectors and accessories was in response to numerous reports of wrong route medication errors where oral or enteral medications were administered IV due to for example failures in tubing connections. A key issue was that syringes were multi-purpose and hence could be used for both the enteral and IV routes. The new design ensures that enteral syringes and accessories cannot be connected to IV lines [15, $25]$, although wrong route administration errors have not been completely eradicated $[26,27]$. Unfortunately, despite the new ENFit design being a major step towards improving patient safety, as discussed at the workshop, its introduction appears to have led to unforeseen difficulties and additional complexities when administering small liquid volumes orally (into the mouth).

The presentations and discussions highlighted the significant resource the pharmaceutical and devices supplier industries spend on the development and testing of oral dosing syringes. The lack of an International Standard (ISO) for oral (and enteral) dosing syringes together with apparently dissimilar regulatory and local requirements make the global design and supply of these devices very challenging. As reflected in the voting, it would be of great benefit to Industry, end-users and Regulators if a globally acceptable standard for oral syringes could be established. Such a standard should be the result of a collaboration between HCPs, drug product developers, device engineers and regulators, and include limits on for example dimensions, design, materials of construction, accuracy, precision and graduations. It would aim to assure that all off-the-shelf oral dosing syringes of specific sizes would have technically and clinically acceptable accuracy and precision and assist in the design of product-specific oral liquid dosing syringes, should they be required. However, it should be remembered that based on the discussions outlined above, bespoke oral measuring devices are not preferred by hospital HCPs and are often discarded. The implementation of global standard design enteral syringes together with medicine bottles with standardised and syringe-compatible bottle neck dimensions and adaptors could also potentially reduce complexity and mitigate risk of dosing errors in the medicine administration process. 
It has been suggested that the minimum acceptable accurately measurable oral liquid volume is $0.2 \mathrm{~mL}[28]$, although this would depend on oral syringe dimensions and physical properties of the liquid such as viscosity. In November 2018, the EMA CHMP adopted and published the new Q\&A developed by the QWP on measuring small volumes referred to during the workshop [29], where it is stipulated that the maximum capacity (size) of a measuring device should be appropriate for the volume to be dosed. It also states that liquid preparations are intended to be administered with a measuring device that is commonly available in the EU and if this is not the case, a dedicated measuring device (with acceptable accuracy and precision) should be co-packaged with the product by the MA holder. Hence, it is important that Industry considers drug product dose volumes that can be accurately measured, to ensure patient and regulatory acceptability of their proposed drug administration strategy. For example, regulatory authorities may consider that the lowest proposed volume for dosing is too small to ensure accuracy, and that this needs to be addressed through either an additional formulation/strength or dedicated dosing device to ensure the medicine's positive benefit to risk profile.

It is recognised that there are some limitations to the outputs of the workshop. For example, many of the participants were identified through EuPFI members and as attendees of previous EuPFI conferences which may have led to some bias. In addition, despite the endeavours of the workshop organisers, the stakeholder groups were not balanced for numbers, no patient representatives attended, and almost half of the participants resided in the UK, all of which may have impacted the scoring of potential solutions.

\section{Conclusions and Workshop Outcomes}

It can be concluded that despite progress being made in the development and supply of paediatric medicines, there are still challenges regarding the supply and use of dosing syringes and enteral accessories for the administration of oral liquids to paediatric patients. The general consensus of workshop participants was that the global harmonisation/standardisation of these devices, as well as medicine bottles and adaptors would mitigate many of the issues faced. In addition, improvement in the dosing accuracy of small liquid volumes is required to reduce the risk of dosing errors, especially in neonates and infants. 
Following the workshop, concerns regarding some of the challenges identified were shared with the ISO Non-active Medical Devices Standards Committee (CEN/TC205). The workshop team have subsequently been advised that the process for the introduction of a new international standard is to submit a New Work Item Proposal (NWIP) to ISO for review and approval. The NWIP should include for example performance requirements (e.g. accuracy, dead space etc.), materials, standardised tip dimensions and position, graduations and units of measure. It is therefore recommended that further engagement and collaboration is sought with key stakeholder groups to further clarify and define practical oral and enteral syringe design and user specifications and seek a way towards achieving standardisation of these devices.

\section{Acknowledgements}

The authors would like to thank Gary Inwards (MHRA) for his assistance and the speakers and participants for their valuable contributions.

\section{References}

1. Charpak N, Ruiz JG, Zupan J, Cattaneo A, Figueroa Z, Tessier R, et al. Kangaroo Mother Care: 25 years after. Acta paediatrica (Oslo, Norway : 1992). 2005 May;94(5):514-22.

2. Commission to the European Parliament and the Council. State of Paediatric Medicines in the EU 10 years of the EU Paediatric Regulation.

https://ec.europa.eu/health/sites/health/files/files/paediatrics/docs/2017 childrensmedicines r eport en.pdf (accessed 28 August 2019)

3. van Riet-Nales DA, de Jager KE, Schobben AF, Egberts TC, Rademaker CM. The availability and age-appropriateness of medicines authorized for children in The Netherlands. British journal of clinical pharmacology. 2011 Sep;72(3):465-73.

4. Wimmer S, Rascher W, McCarthy S, Neubert A. The EU paediatric regulation: still a large discrepancy between therapeutic needs and approved paediatric investigation plans. Paediatr Drugs 2014 Oct;16(5):397-406.

5. Ruggieri L, Giannuzzi V, Baiardi P, Bonifazi F, Davies EH, Giaquinto C, et al. Successful privatepublic funding of paediatric medicines research: lessons from the EU programme to fund research into off-patent medicines. European journal of pediatrics. 2015 Apr;174(4):481-91.

6. O'Brien F, Clapham D, Krysiak K, Batchelor H, Field P, Caivano G, Pertile M, Nunn A, Tuleu C. Making Medicines Baby Size: The Challenges in Bridging the Formulation Gap in Neonatal Medicine. Int. J. Mol. Sci. 2019;20: 2688. 
7. Allegaert K, van den Anker J. Neonatal drug therapy: The first frontier of therapeutics for children. Clinical pharmacology and therapeutics. 2015 Sep;98(3):288-97.

8. Lau C. Development of Suck and Swallow Mechanisms in Infants. Annals of nutrition \& metabolism. 2015;66 Suppl 5:7-14.

9. Ainscough LP, Ford JL, Morecroft CW, Peak M, Turner MA, Nunn AJ, et al. Accuracy of intravenous and enteral preparations involving small volumes for paediatric use: a review. European journal of hospital pharmacy : science and practice. 2018 Mar;25(2):66-71.

10.Tanner S, Wells M, Scarbecz M, McCann BW, Sr. Parents' understanding of and accuracy in using measuring devices to administer liquid oral pain medication. Journal of the American Dental Association (1939). 2014 Feb;145(2):141-9.

11.Dockhorn S, Feuersenger D, Schuenemann S, Knauf B, Duerr S, Schubert-Zsilavecz M, et al. Study of microbial contamination and dosing accuracy of oral dispensers. Journal of clinical pharmacy and therapeutics. 2010 Jun;35(3):279-87.

12.European Medicines Agency Committee for Medicinal Products for Human Use. Guideline on pharmaceutical development of medicines for paediatric use. 2013 https://www.ema.europa.eu/en/documents/scientific-guideline/guideline-pharmaceuticaldevelopment-medicines-paediatric-use en.pdf (accessed 2 August 2019).

13. Walsh J, Math MC, Breitkreutz J, Zerback T, Wachtel H. Devices for oral and respiratory paediatric medicines: What do healthcare professionals think? International journal of pharmaceutics. 2015 Aug 15;492(1-2):304-15.

14.Guenter P, Lyman B. ENFit Enteral Nutrition Connectors. Nutrition in clinical practice : official publication of the American Society for Parenteral and Enteral Nutrition. 2016 Dec;31(6):769-72.

15.Salunke S, Liu F, Batchelor H, Walsh J, Turner R, Ju TR, et al. European Paediatric Formulation Initiative (EuPFI)-Formulating Ideas for Better Medicines for Children. AAPS PharmSciTech. 2017 Feb;18(2):257-62.

16. Arenas-Lopez S, Gurung K, Tibby SM, Calleja Hernandez MA, Tuleu C. Accuracy of enteral syringes with commonly prescribed paediatric liquid medicines. Archives of disease in childhood. 2017 Jul;102(7):655-9.

17. Richey RH, Hughes C, Craig JV, Shah UU, Ford JL, Barker CE, et al. A systematic review of the use of dosage form manipulation to obtain required doses to inform use of manipulation in paediatric practice. International journal of pharmaceutics. 2017 Feb 25;518(1-2):155-66.

18. Sobhani P, Christopherson J, Ambrose PJ, Corelli RL. Accuracy of oral liquid measuring devices: comparison of dosing cup and oral dosing syringe. The Annals of pharmacotherapy. 2008 Jan;42(1):46-52. 
19. European Medicines Agency Committee for Medicinal Products for Human Use. Reflection Paper: formulations of choice for the paediatric population. 2006

https://www.ema.europa.eu/en/documents/scientific-guideline/reflection-paper-formulationschoice-paediatric-population en.pdf (accessed 2 August 2019).

20.Joshi P, Bavdekar SB. Liquid Drug Dosage Measurement Errors with Different Dosing Devices. Indian journal of pediatrics. 2019 Apr;86(4):382-5.

21.Duffett L. Patient engagement: What partnering with patient in research is all about. Thrombosis research. 2017 Feb;150:113-20.

22.Stegemann S, Ternik RL, Onder G, Khan MA, van Riet-Nales DA. Defining Patient Centric Pharmaceutical Drug Product Design. The AAPS journal. 2016 Sep;18(5):1047-55.

23. Medicines and Healthcare products Regulatory Agency. Human Factors and Usability Engineering - Guidance for Medical Devices Including Drug-device Combination Products, 2017 https://assets.publishing.service.gov.uk/government/uploads/system/uploads/attachment data/ file/645862/HumanFactors Medical-Devices v1.0.pdf (accessed 28 August 2019).

24.Ryu GS, Lee YJ. Analysis of Liquid Medication Dose Errors Made by Patients and Caregivers Using Alternative Measuring Devices. J Manag Care Spec Pharm 2012 Jul;18(6):439-445.

25.Anderson L. ENFit: a major patient safety breakthrough. British journal of community nursing. 2016 Jun-Jul;Suppl Nutrition:S7-8.

26. Grissinger M. A Successful ENFit Launch Still Won't Stop All Incidents of Oral Medications Given Intravenously. P \& T : a peer-reviewed journal for formulary management. 2018 Jul;43(7):379-80.

27.Independent Report of the Healthcare Safety Investigation Branch. Inadvertent administration of an oral liquid medicine into a vein, April 2019 https://www.hsib.org.uk/investigationscases/inadvertent-administration-oral-liquid-medicine-vein/final-report/(accessed 28 August 2019)

28. Rawlence E, Lowey A, Tomlin S, Auyeung V. Is the provision of paediatric oral liquid unlicensed medicines safe? Archives of disease in childhood Education and practice edition. 2018 Dec;103(6):310-3.

29.European Medicines Agency Quality of Medicines Questions and Answers: Part 2 Specific types of product - Graduation of measuring devices for liquid dosage forms - Updated Nov. 2018 https://www.ema.europa.eu/en/human-regulatory/research-development/scientificguidelines/qa-quality/quality-medicines-questions-answers-part-2\#specific-types-of-product---graduation-of-measuring-devices-for-liquid-dosage-forms---updated-nov.-2018-section (accessed 28 August 2019) 